\title{
A SPLITTING THEOREM FOR MANIFOLDS WITH INVOLUTION AND TWO APPLICATIONS
}

\author{
REINHARD SCHULtz*
}

One of the most basic questions of differential topology is the extent to which the geometrical properties of smooth manifolds are invariant under homeomorphism or homotopy equivalence. Here are two specific questions of this type:

\begin{abstract}
Splitting problem. Given a closed smooth manifold $M$ with a splitting of $M$ as a union $A \cup_{C} B$ of two manifolds with common boundary $C$, let $f: M^{\prime} \rightarrow M$ be a homotopy equivalence. Is there a splitting of $M^{\prime}$ into $A^{\prime} \cup_{C^{\prime}} B^{\prime}$ such that $f$ maps $A^{\prime}$ to $A, B^{\prime}$ to $B$, and $C^{\prime}$ to $C$ by homotopy equivalences?
\end{abstract}

Invariance of tangent bundles. Given a homotopy equivalence of closed smooth manifolds $f: M \rightarrow N$, to what extent are the tangent sphere bundles of $M$ and $N$ equivalent?

Substantial results on both questions were obtained during the nineteen sixties, and further work was done during the following decade. For example, if in the first question we assume that all manifolds under consideration are simply connected and of sufficiently high dimension, then a splitting of the prescribed type always exists (compare [24], page 133). In fact, stronger results can also be proved if the fundamental groups of $A, B, C$ are interrelated in a suitable manner (compare Wall [24], Chapter 12A, or Cappell [5]).

In the second question one cannot expect the sphere bundles to be linearly or even topologically equivalent. However, a result of Atiyah [1] shows that the tangent sphere bundles are stably fiber homotopy equivalent, and a result of R. Benlian and J. Wagoner [3] shows that these bundles are already fiber homotopy equivalent before stabilization. Although this result is basically a statement in homotopy theory, the original proof in [3] was highly geometric, relying heavily on Wall's codimension one splitting theorem in [24]; subsequent research produced an intrinsically homotopy-theoretic proof (see Dupont [12] and Sutherland [21]). In this paper we shall show that the basic geometric argument in [3] has an equivariant analog. In particular, we shall prove a splitting theorem for $\mathbb{Z}_{2}$-homotopy equivalences under general position assumptions slightly weaker than the Gap Hypothesis of [15]. Using this result we shall show that the tangent sphere bundles of homotopy equivalent manifolds are $\mathbb{Z}_{2}$-equivariantly fiber homotopy equivalent, where $\mathbb{Z}_{2}$ acts by the fiberwise antipodal map. Previous results of S. Straus [19] and M. Crabb [6] (Proposition 1.1 , pages $2-3$ ) show that these bundles with involution are stably $\mathbb{Z}_{2}$-fiber homotopy equivalent.

*Partially supported by $\quad$ NSF $\quad$ Grant $\quad$ MCS86-02543 
In fact, [19] contains an attempt to prove slightly weaker versions of the unstable results mentioned above, but there is a gap in the argument.

Section 1 furnishes a proof of the following splitting theorem:

Theorem A. Let $N^{2 k}$ be a closed, 1-connected smooth $\mathbb{Z}_{2}$-manifold such that $k \geq 3$ and the fixed point set $N_{0}$ is a 1-connected closed $k$-manifold. Assume that $N$ admits an invariant decomposition $A \cup_{C} B$, where $A$ and $B$ are compact smooth $\mathbb{Z}_{2}$-manifolds with $C=\partial A=\partial B$, the fixed point set $N_{0}$ is contained in the interior of $A$, and $A, B$, and $C$ are all 1 -connected. If $N^{*}$ is another closed smooth $\mathbb{Z}_{2}$-manifold and $f: N^{*} \rightarrow N$ is a $\mathbb{Z}_{2}$-homotopy equivalence, then there is an invariant splitting $N^{*}=A^{*} \cup B^{*}$ such that $f$ induces a $\mathbb{Z}_{2}$-homotopy equivalence of triads from $(N ; A, B)$ to $\left(N^{*} ; A^{*}, B^{*}\right)$.

In [19] S. Straus proved an analogous result assuming that the dimension of $N_{0}$ is less than half the dimension of $N$ but with no hypothesis on the fundamental group of $N$. This result is actually one of the first uses of the Gap Hypothesis in applying surgery to manifolds with group actions. Recently W. Browder and K. H. Dovermann have obtained variants of Straus' result and applied these to analyze equivariant degree 1 maps of $G$-manifolds.

A nonequivariant version of Theorem A with minimal assumptions on fundamental groups is due to Wall (see [24], Theorem 12.1, page 133). In the case under consideration it is necessary to combine Wall's techniques with some results of Dovermann on $\mathbb{Z}_{2}$-surgery ([8]; also see [10]). The latter requires simple connectivity assumptions, and this is the reason for the simple connectivity condition in Theorem A. Of course, it is natural to ask if Theorem A holds under weaker assumptions on fundamental groups. This and other questions arising in this paper will be discussed at the end of Section 2. That section also contains the result on tangent sphere bundles (Theorem 2.1). We shall also use the splitting theorem to interpret a result of Dovermann on $\mathbb{Z}_{2}$-surgery in terms of fixed point data. Specifically, suppose we are given a $\mathbb{Z}_{2}$-surgery problem into a simply connected $(4 k+2)$-manifold whose fixed point set is 1 -connected and $(2 k+1)$-dimensional. Then in many cases the $\mathbb{Z}_{2}$-surgery obstruction is determined by the ordinary Kervaire invariant and the difference between certain fixed point data for the domain and codomain (see Theorem 2.5). This is formally analogous to an interpretation of the $\mathbb{Z}_{2}$-surgery obstruction in the $4 k$-dimensional case (with 2k-dimensional fixed point set) that follows from [8], [10] and the Atiyah-Singer $G$-signature formula; further details appear in Section 2.

\section{Acknowledgments}

I am grateful to W. Browder and K. H. Dovermann for their willingness to discuss their unpublished results involving equivariant codimension 1 splittings. Also, I am grateful to J. C. Becker and M. Mahowald for helpful conversations. Finally, the University of California's hospitality to the 1986 International Congress of Mathematicians in Berkeley also played a significant role, for this provided me the opportunity to find Straus' interesting but (apparently) little known thesis.

\section{Proof of Theorem A}

Let $N_{0}^{*}$ be the fixed point set of $\mathbb{Z}_{2}$ on $N^{*}$. Then $f$ maps $N_{0}^{*}$ to $N_{0}$, and therefore $f^{-1}(\partial A) \cap N_{0}^{*}=$ $\phi$. It follows that $f$ is equivariantly homotopic to a map $g$ such that $g$ is transverse to $\partial A$ and $g^{-1}(\partial A) \cap N_{0}^{*}=\phi$; it follows that $N_{0}^{*}$ is contained in $g^{-1}(A)$ and $g$ induces a map of triads

$$
\left(g: g_{A}, g_{B}\right):\left(N^{*} ; g^{-1}(A), g^{-1}(B)\right) \rightarrow(N ; A, B) .
$$

Wall's $\pi-\pi$ Theorem ([2], [4], Chapter 4) implies that the map $g_{B}$ of free $\mathbb{Z}_{2}$ manifolds is $\mathbb{Z}_{2^{-}}$ normally cobordant to a $\mathbb{Z}_{2}$-homotopy equivalence $h_{B}$. By an extension argument as in [24], pages 
134, lines $1-2$, the map of triads $\left(g ; g_{A}, g_{B}\right)$ is normally cobordant as a map of triads to some $\left(h ; h_{A}, h_{B}\right)$. In fact, we can and shall insist that the cobordism is a product $V \times[0,1]$ on some neighborhood $V$ of $N_{0}^{*}$.

Consider now the map $h_{A}$; this is an equivariant homotopy equivalence on the boundary, and therefore $h_{A}$ satisfies the criteria to define a $\mathbb{Z}_{2}$-surgery problem satisfying the Borderline Gap Hypothesis (compare [8] or [10]). The results of these papers show that $h_{A}$ can be surgered equivariantly to a $\mathbb{Z}_{2}$-homotopy equivalence, with the boundary left untouched and all surgeries disjoint from the fixed point set, if and only if an obstruction in a certain Witt group vanishes. Since $h_{B}$ is an equivariant homotopy equivalence and everything in sight is simply connected, it is elementary to verify that the equivariant surgery obstructions for $h$ and $h_{A}$ are equal. But $h$ is $\mathbb{Z}_{2}$-normally cobordant to the $\mathbb{Z}_{2}$-homotopy equivalence $f$, and therefore the obstruction in question must vanish. Let $h_{A}^{*}$ be the $\mathbb{Z}_{2}$-homotopy equivalence normally cobordant to $h_{A}$ such that the above-mentioned boundary and fixed point conditions are satisfied. If $h^{*}=h_{A}^{*} \cup h_{B}$, then $h^{*}$ defines a $\mathbb{Z}_{2}$-homotopy equivalence of triads $\left(h^{*} ; h_{A}^{*}, h_{B}\right)$, and $h^{*}$ is $\mathbb{Z}_{2}$-normally cobordant to $f$.

Let $W$ be the domain of the $\mathbb{Z}_{2}$-normal cobordism between $f$ and $h^{*}$. We claim it is possible to perform $\mathbb{Z}_{2}$-surgery on $W$, leaving the boundary and fixed point set untouched, to obtain a $\mathbb{Z}_{2}$-homotopy equivalence $W^{\prime} \rightarrow N \times[0,1]$. If this is possible, then Theorem A follows, for $W^{\prime}$ is an equivariant $h$-cobordism and a theorem of Rothenberg [16] (Theorem 3.4, page 291) combined with $W h\left(\mathbb{Z}_{2}\right)=0$ shows that $W^{\prime}$ is $\mathbb{Z}_{2}$-diffeomorphic to $N^{*} \times[0,1]$.

By construction $W$ is obtained by adding handles to $N^{*} \times[0, \varepsilon]$ away from the fixed point set. Therefore by general position we can perform equivariant surgery on $W$, away from the boundary and the fixed point set, to obtain a similarly structured $\mathbb{Z}_{2}$-normal cobordism $W_{0}$ that is highly connected. Specifically, the first nonzero surgery kernel for the $(2 k+1)$-manifold $W_{0}$ arises in dimension $k$. Take a set of generators for this kernel over $\mathbb{Z}\left[\mathbb{Z}_{2}\right]$ and represent them by embedded $k$-spheres in Int $W_{0}-F i x\left(W_{0} ; \mathbb{Z}_{2}\right)$. Exactly as in Chapter 6 of Wall's book [24], these embeddings determine a pair of subkernels in the standard hyperbolic $(-1)^{k}$-Hermitian form over $\mathbb{Z}\left[\mathbb{Z}_{2}\right]$. From this pair one obtains an invertible matrix $\alpha$ over $\mathbb{Z}\left[\mathbb{Z}_{2}\right]$ that preserves the hyperbolic form; denote the group of such matrices by $S U^{\langle k\rangle}$. As in Wall [24], Chapter 6, the matrix $\alpha \in S U^{\langle k\rangle}$ depends on various choices and is not uniquely defined. However, since the embedded $k$-spheres in $W_{0}$ are disjoint from the fixed point set, the analysis of Wall [24], Chapter 6, goes through and shows that surgery can be completed, leaving the fixed point set and boundary untouched, if the equivalence class of $\alpha$ under the projection

$$
S U^{\langle k\rangle} \rightarrow L_{2 k+1}\left(\mathbb{Z}_{2},(-1)^{k}\right)
$$

(as defined in [24], page 65) is zero. But it is known that the $L$-groups in question are always zero (see [24], Theorem 13.A.1, page 162). Therefore one can do surgery on $W_{0}$ away from the boundary and fixed set to obtain a $\mathbb{Z}_{2}$-homotopy equivalence into $N \times[0,1]$. As noted previously, this suffices to complete the proof

Complement to Theorem A. A similar result holds if we allow the fixed point set to be the disjoint union of a 1-connected closed $k$-manifold $N_{1}$ and a union $N_{2}$ of lower dimensional submanifolds.

Notice that the dimensions of all fixed point components are congruent mod 2 because the mod 2 codimension of the fixed point set determines whether the action preserves or reverses orientation (assuming the fixed point set is nonempty).

This follows because one can always choose an embedding of a manifold of dimension $\leq k$ in $N$ to be disjoint from $N_{2}$. For surgery theory one needs to use embedded spheres of these dimensions, 
and consequently all the embedded spheres needed for surgery theory can be chosen to miss $N_{2}$. Likewise, if one has an embedding of cobordism triads

$$
N_{2} \times([0,1] ;\{0\},\{1\}) \rightarrow\left(W_{2 k+1} ; \partial_{0} W, \partial_{1} W\right),
$$

then $\operatorname{dim} N_{2} \leq k-2$ implies that embeddings of $(k+1)$-manifolds in $W$ may be made disjoint from $W_{2} \times[0,1]$. With this understood the proof of Theorem A goes through.

\section{Special Cases And applications}

Given a closed smooth manifold $X$, the map $T_{X}\left(x_{1}, x_{2}\right)=\left(x_{2}, x_{1}\right)$ defines a smooth involution on $X \times X$ whose fixed point set is the diagonal. Given a homotopy equivalence $f: M \rightarrow N$ between two closed smooth manifolds, the map

$$
f \times f: M \times M \rightarrow N \times N
$$

defines an equivariant homotopy equivalence. If we apply the Splitting Theorem of Section 1 to $f \times f$ (assuming $\pi_{1}(M)=\{1\}$ ), we obtain the following conclusion:

Theorem 2.1. Let $f: M \rightarrow N$ be a homotopy equivalence as above with $M$ and $N$ simply connected. Let $S\left(\tau_{M}\right)$ and $S\left(f^{*} \tau_{N}\right)$ denote the unit sphere bundles of the vector bundles obtained from the tangent bundle $\tau_{M}$ of $M$ and the pullback of the tangent bundle of $\tau_{N}$; let $\mathbb{Z}_{2}$ act freely on each sphere bundle via multiplication by -1 . Then $S\left(\tau_{M}\right)$ and $S\left(f^{*} \tau_{N}\right)$ are $\mathbb{Z}_{2}$-fiber homotopy equivalent.

Remarks. As noted before, previously published results show that the bundles are fiber homotopy equivalent (but not necessarily equivariantly so) and stably $\mathbb{Z}_{2}$-fiber homotopy equivalent. However, stabilization for spherical fibrations with fiberwise antipodal involutions is intrinsically more complicated than stabilization of vector bundles or ordinary spherical fibrations (compare [17]; a result of H. Toda [23] (see 11.7) shows that the stabilization theories in the latter cases are very closely related).

Proof. (Following [19], Section 6) If $X$ is an unbounded smooth manifold and $\mathbb{Z}_{2}$ acts on $X \times X$ via the involution $T_{X}$, then the diagonal is the fixed point set and the equivariant normal bundle of the diagonal is $\mathbb{Z}_{2}$-isomorphic to the tangent bundle of $X$ with the antipodal involution. This yields a $\mathbb{Z}_{2}$-equivariant splitting

$$
X \times X=D\left(\tau_{X}\right) \cup_{\partial} D P(X),
$$

where the interior of $D P(X)$ is $\mathbb{Z}_{2}$-diffeomorphic to $X \times X-\Delta_{X}$, where $\Delta_{X}$ is the diagonal. Furthermore, if $V$ is an invariant neighborhood of $\Delta_{X}$ in $X \times X$, one can choose the splitting so that $\Delta_{X}$ lies in the interior of $V$.

Given the homotopy equivalence $f$, consider the equivariant homotopy $f \times f$ and the equivariant splitting of $N \times N$ into $D\left(\tau_{N}\right) \cup D P(N)$. Therefore Theorem A implies that $f \times f$ splits up to homotopy as a $\mathbb{Z}_{2}$-homotopy equivalence of triads

$$
(M \times M ; P, Q) \rightarrow\left(N \times N ; D\left(\tau_{N}\right), D P(N)\right) .
$$

Let $W=\partial P=\partial Q$. As in the preceding paragraph, we may assume that $D\left(\tau_{M}\right) \subseteq \operatorname{Int}(P)$. It follows that

$$
M \times M-\left(\operatorname{Int} D\left(\tau_{M}\right) \cup \operatorname{Int} D P(M)\right)
$$


is a $\mathbb{Z}_{2}$-equivariant $h$-cobordism of free $\mathbb{Z}_{2}$-manifolds. Therefore by the $h$-cobordism theorem and the vanishing of $W h\left(\mathbb{Z}_{2}\right)$ we conclude that $W$ is $\mathbb{Z}_{2}$-equivariantly diffeomorphic to $S\left(\tau_{M}\right)$. This yields a homotopy commutative diagram

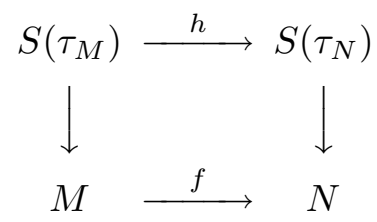

in which $h$ is $\mathbb{Z}_{2}$-equivariant. Therefore we can apply an equivariant version of Dold's recognition principle for fiber homotopy equivalences ([7]; see Waner [25] for the equivariant version), concluding that $S\left(\tau_{M}\right)$ and $S\left(f^{*} \tau_{N}\right)$ are $\mathbb{Z}_{2}$-fiber homotopy equivalent

Corollary 2.2. Let $M$ and $N$ be closed 1-connected $n$-manifolds $(n \geq 3)$. Then the open deleted products $M \times M-\Delta_{M}$ and $N \times N-\Delta_{N}$ are $\mathbb{Z}_{2}$-homotopy equivalent.

Proof. ¿From the proof of 2.1 we see that $D P(M)$ and $D P(N)$ are $\mathbb{Z}_{2}$-homotopy equivalent, and by the collar neighborhood theorem the same is true for their interiors. But the interior of $D P(X)$ is $\mathbb{Z}_{2}$-diffeomorphic to $X \times X-\Delta_{X}$

Remarks. For each positive integer $n$ the space $D^{n} \times D^{n}-\Delta\left(D^{n}\right)$ is $\mathbb{Z}_{2}$-homotopy equivalent to $S^{n-1}$ with the antipodal involution and therefore the $\mathbb{Z}_{2}$-homotopy type of the $\mathbb{Z}_{2}$-space $X \times X-\Delta_{X}$ is not a homotopy invariant for $X$ a finite complex. Thus some restriction to manifolds of the same dimension is needed for results such as 2.2. One can use results of J. Hodgson to define an analog of the $\mathbb{Z}_{2}$-space $X \times X-\Delta_{X}$ when $X$ is a Poincaré complex (see [26], for example). However, one needs a result such as Theorem A to conclude that Hodgson's complex is $\mathbb{Z}_{2}$-homotopy equivalent to $M \times M-\Delta_{M}$ for $M$ a closed manifold.

The same sort of argument as in 2.1 proves the following result:

Theorem 2.3. Let $f: M^{2 k} \rightarrow N^{2 k}$ be a $\mathbb{Z}_{2}$-homotopy equivalence of closed, smooth, 1-connected $\mathbb{Z}_{2}$-manifolds. Assume that the fixed point sets are 1 -connected and that $k \geq 3$. Let $\alpha(M)$ and $\alpha(N)$ denote the equivariant normal bundles of the fixed point sets, and let $f_{0}$ denote the induced map of fixed point sets. Then the sphere bundles $S(\alpha(M))$ and $S\left(f_{0}{ }^{*} \alpha(N)\right)$ are $\mathbb{Z}_{2}$-fiber homotopy equivalent.

Proof. (Sketch) Split $N$ as a union of $D(\alpha(N))$ and $N^{*}$, and approximate $f$ by an equivalence of triads

$$
(M ; P, Q) \rightarrow\left(N ; D(\alpha(N)), N^{*}\right) .
$$

Let $W=\partial P=\partial Q$. Then $D(\alpha(M))$ lies in the interior of $P$, and the region between $W$ and $S(\alpha(M))$ is an equivariant $h$-cobordism. If we let $M_{0}$ and $N_{0}$ denote the fixed point sets of $M$ and $N$, it follows that there is a commutative diagram

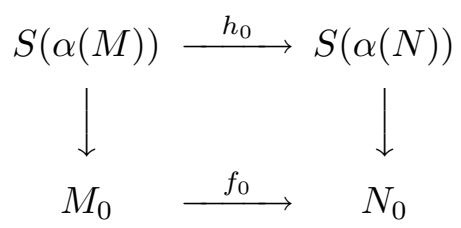

analogous to the diagram in the proof of 2.1. The same considerations as in the latter result show that $h_{0}$ determines a $\mathbb{Z}_{2}$-equivariant fiber homotopy equivalence from $S(\alpha(M))$ to $S\left(f_{0}^{*} \alpha(N)\right)$ 
Theorem 2.3 yields a simple geometric interpretation for one of Dovermann's $\mathbb{Z}_{2}$-surgery obstructions [8] in a large number of cases. We shall assume we are dealing with smooth $\mathbb{Z}_{2}$-surgery problems of the form

$$
h: M^{2 k} \rightarrow X^{2 k}
$$

where $k$ is odd and the fixed point sets are closed, 1-connected, $k$-dimensional manifolds for $k \geq 5$ (but $k \neq 7$ ). Furthermore, we shall assume that $h$ induces a homotopy equivalence of fixed point sets; this induced map will be written as $h_{0}: M_{0} \rightarrow X_{0}$.

The results of [8], [10] show that the $\mathbb{Z}_{2}$-surgery obstruction for $h$ lies in $\mathbb{Z}_{2} \oplus \mathbb{Z}_{2}$, where the first summand is detected by the ordinary Kervaire invariant and the second by the mod 2 rank invariant of some surgery kernel. If $X$ is a linear sphere and the bundle data for the problem involves the trivial $\mathbb{Z}_{2}$-vector bundle over $X$, then fairly standard considerations show that the mod 2 rank invariant is 1 if the normal bundles $\alpha(M), \alpha(X)$ are not isomorphic and 0 if they are. Using Theorem 2.3 we shall prove a similar result for a large class of choices for $X$.

If $k \neq 1,3,7$ is an odd positive integer and $Y$ is a closed 1 -connected, $k$-manifold, then a well-known argument (compare James and Thomas [14]) yields an exact sequence of pointed sets

$$
\mathbb{Z}_{2} \stackrel{\omega}{\rightarrow}\left[Y, B S O_{k}\right] \rightarrow \widetilde{K O}(Y)
$$

in which $\omega$ comes from an action of $\mathbb{Z}_{2}$ on the middle set sending a class represented by $\xi$ to

$$
Y \stackrel{\text { pinch }}{\longrightarrow} Y \vee S^{k} \stackrel{\xi \vee \tau}{\longrightarrow} B S O_{k}
$$

where $\tau: S^{k} \rightarrow B S O_{k}$ classifies the tangent bundle for $S^{k}$. If $Y=S^{k}$ this action is free, but there are cases which the action is not free, and in fact for fixed $Y$ the action can be free on some orbits and trivial on others. For example, if $Y=\mathbb{R} P^{k}$. with $k$ odd and $k+1$ not a power of 2 , then there is only one $k$-plane bundle stably equivalent to the tangent bundle of $Y$ but there are two nonisomorphic $k$-plane bundles that are stably isomorphic to the normal bundle of $Y$ in some large Euclidean space (see James and Thomas [15]; the first result is given in Corollary 1.11, page 490, and the second in Corollary 1.10). In fact results of W.A. Sutherland show that the associated $(k-1)$-sphere bundles are not even fiber homotopy equivalent in the latter case (see [20]; a generalization to arbitrary oriented $k$-manifolds is given in [22]).

Definition. Let $k$ be an odd integer, $V^{k}$ a closed $k$-manifold, and $\xi$ a vector bundle over $V$. Following Sutherland [22] we define the fiber homotopy James-Thomas number of $\xi$ to be the number of fiber homotopy equivalence classes of linear $(k-1)$-sphere bundles that are stably fiber homotopy equivalent to $\xi$ (by "linear" we mean the unit sphere bundle of a $k$-dimensional vector bundle). The $k$-connectivity of $B O_{k} \rightarrow B O$ and $B G_{k} \rightarrow B G$ together with the preceding discussion show that this number if 1 or 2 . We shall denote its value by $j(\xi)$. If $\xi$ is stably fiber homotopically trivial then the results of [11] and [12] imply $j(\xi)=2$. A result of Sutherland [20] gives a means for computing $j(\xi)$ in general and leads to exact evaluations in many interesting cases (compare [20], [22]).

Let $V^{k}$ and $\xi$ be as above, and assume $j(\xi)=2$. We shall say that $(V, \xi)$ satisfies the Dupont condition if all $k$-dimensional polynomials in the Stiefel-Whitney classes of $\nu-\xi$ are zero, where $\nu$ is the stable normal bundle of $V$. As noted in [12], the tangent bundle of $V$, satisfies this condition. More generally, if the image of $\xi-\nu$ in $\widetilde{J O}(V)$ is divisible by 2, then the argument of [12], Lemma 4.2 , page 209, and the remark at the top of page 210 (in [12]) shows that the Dupont condition is satisfied. 
Suppose now that $(V, \xi)$ satisfies the Dupont condition and $j(\xi)=2$, and let $\eta$ be a $k$-plane bundle that is stably fiber homotopically equivalent to $\xi$. In this case the results of J. Dupont [12] define an invariant $b(\eta) \in \mathbb{Z}_{2}$ (the Browder-Dupont invariant) such that $b$ distinguishes the two classes of linear $(k-1)$-spherical fibrations (see in particular [12], Corollary 4.5, page 211). In this case the action of $\mathbb{Z}_{2}$ on $\left[V, B O_{k}\right]$ adds 1 to the Browder-Dupont invariant; it follows that there are two linear isomorphism classes of $k$-plane bundles that are stably isomorphic to $\eta$, and these can be distinguished by the fiber homotopy types of the associated $(k-1)$-sphere bundles.

The following is an immediate consequence of the discussion above and Theorem 2.3.

Proposition 2.4. Let $k \geq 3$ be odd, and let $f: M^{2 k} \rightarrow N^{2 k}$ be a $\mathbb{Z}_{2}$-homotopy equivalence of closed, smooth, 1-connected $\mathbb{Z}_{2}$-manifolds. Assume that the fixed point sets are 1-connected $k$ manifolds with equivariant normal bundles $\alpha(M)$ and $\alpha(N)$ respectively. Furthermore, assume that $\alpha(N)$ and $\alpha(M)$ satisfy $j(\alpha)=2$ and the condition on Stiefel-Whitney classes needed to define the Browder-Dupont invariant. Then $b(\alpha(M))$ and $b(\alpha(N))$ are equal.

Proof. By Theorem 2.3 the $(k-1)$-sphere bundles of $\alpha(M)$ and $\alpha(N)$ are fiber homotopically equivalent, and in fact $\mathbb{Z}_{2}$-equivariantly so. Therefore the Browder-Dupont invariants are equal if they are meaningful. But we have added the assumptions necessary to define this invariant

This result yields a geometric interpretation of a $\mathbb{Z}_{2}$-surgery obstruction defined in work of Dovermann ([8], Section 3; see also [10], discussion preceding Theorem B). In particular, one can restate the central result of the theory as follows.

Theorem 2.5. Let $f: X \rightarrow Y$ come from a suitably defined $\mathbb{Z}_{2}$-normal map of smooth $(4 k+2)$ dimensional $\mathbb{Z}_{2}$-manifolds, where $f$ induces a homotopy equivalence of fixed point sets and the latter are 1-connected. Assume that the Browder-Dupont invariants are definable for $\alpha(X)$ and $\alpha(Y)$. Then $f$ is normally cobordant to a $\mathbb{Z}_{2}$-homotopy equivalence, relative to the fixed point sets, if and only if

(i) the ordinary Kervaire invariant vanishes,

(ii) $b(\alpha(X))=b(\alpha(Y))$.

Remarks. 1. The technical definition of a $\mathbb{Z}_{2}$-normal map involves a $\mathbb{Z}_{2}$-equivariant map $f$ and some other data that are not needed in the present discussion. For the precise definitions see [10].

2. As in the preceding discussion we let $\alpha(W)$ denote the equivariant normal bundle of the fixed point set of $W$.

3. This result strongly resembles the conclusion in the $4 k$-dimensional case, where the obstructions to $\mathbb{Z}_{2}$-surgery can be viewed as the ordinary signature and the difference between selfintersection invariants of the fixed point sets (compare [8], Section 2, and [10], Theorems $A_{0}$ and $A_{2}$; this uses the Hirzebruch formula for the signature of an involution given by [13], Proposition 6.15, page 583, and [2], Abschnitt 4. Since the Dupont invariant can be viewed as a mod two self-intersection invariant of sorts, Theorem 2.5 essentially states that the $\mathbb{Z}_{2}$-surgery obstructions in the $(4 k+2)$-dimensional case are the ordinary simply connected surgery obstruction and the difference between mod 2 intersection invariants for the fixed point sets.

Proof of 2.5. The results of Dovermann [8] show that the surgery obstructions for $f$ are given by the ordinary Kervaire invariant and the mod 2 rank of the surgery kernel for a highly connected modification of $f$; call the latter invariant $r(f)$. Let $\Delta b(f)$ denote $b(\alpha(X))-b(\alpha(Y))$. It will suffice to prove that if $f$ satisfies the hypotheses of the theorem and has trivial Kervaire invariant, then $r(f)=0$ if and only if $\Delta b(f)=0$. 
Suppose that $r(f)=0$. Then $f$ is normally cobordant to a $\mathbb{Z}_{2}$-homotopy equivalence such that the cobordism of fixed point sets is a product of either end with [0,1] (see [8], Section 4, or [10], Theorem B). Therefore 2.4 implies that $b(\alpha(X))=b(\alpha(Y))$, and hence $\Delta b(f)=0$.

Conversely, suppose $r(f)=1$. Let $g: S^{k} \times S^{k} \rightarrow S^{2 k}$ be the degree 1 map. If we let $\mathbb{Z}_{2}$ act linearly on $S^{2 k}$ with $S^{k}$ as a fixed point set and take the involution on $S^{k} \times S^{k}$ that reverses coordinates, then $g$ is homotopic to an equivariant map and in fact defines a $\mathbb{Z}_{2}$-surgery problem (compare [9], or [18]). As in [9],[18] we can form the equivariant connected sum of $f$ and $g$ along the fixed point set. Since $\alpha\left(S^{k} \times S^{k}\right)$ is the tangent bundle $\tau$ to $S^{k}$ and $b(\tau)=1$, it is a straightforward exercise to calculate that

$$
b\left(\alpha\left(X \# S^{k} \times S^{k}\right)\right)=b(\alpha(X))+1 .
$$

Therefore we have $\Delta b(f \# g)=\Delta b(f)+1$. But it is also immediate that $r(f \# g)=r(f)+1$. Since $r(f)=1$ this means $r(f \# g)=0$, and therefore $\Delta b(f \# g)=0$ by the preceding paragraph. But $\Delta b(f \# g)=\Delta b(f)+1$ now implies $\Delta b(f)=1$

Final Remarks

I. Applications to isovariant approximation. As noted in the introduction there are codimension one splitting theorems like Theorem A if the Gap Hypothesis holds. In other words, if we stratify a $G$-manifold by invariant connected submanifolds, each with one orbit type, then the dimension of each stratum is greater than twice the dimensions of the lower strata. For $G=\mathbb{Z}_{p}$ ( $p$ prime) there are results of this type in S. Straus' thesis [19] (see Theorems 5.1 and 5.3). Recently K. H. Dovermann [27] and W. Browder [4] have independently discovered further results of this type and used them to study isovariant approximations to equivariant maps (an equivariant map is isovariant if it preserves isotropy subgroups). In particular, Browder has shown that a $G$-homotopy equivalence is $G$-homotopic to an isovariant map if the Gap Hypothesis holds and certain lowdimensional problems are avoided. Theorem A implies a similar isovariant approximation result for $\mathbb{Z}_{2}$-homotopy equivalences of closed 1 -connected $\mathbb{Z}_{2}$-manifolds of even dimension (say $2 k$ ), provided the fixed point set is a union of a 1-connected $k$-manifold and components of strictly lower dimension.

II. Removing hypotheses on fundamental groups. The results of this paper all require simple connectivity. It is natural to ask if the main results of this paper hold for $\mathbb{Z}_{2}$-manifolds with more general fundamental groups. Among other things this would require an extension of Dovermann's techniques to other fundamental groups.

III. Alternate approaches. Since Theorem 2.1 has a homotopy-theoretic hypothesis and conclusion, it would be desirable to have an alternate proof that did not require the use of surgery theory.

\section{REFERENCES}

1. M. F. Atiyah, Thom complexes, Proc. London Math. Soc. (3) 11 (1961), 741-752.

2. The index of elliptic operators III, Ann. of Math. 87 (1968), 546-604.

3. R. Benlian and J. Wagoner, Type d'homotopie et réduction structurale des fibrés vectoriels, C. R. Acad. Sci. (Paris) Sér. A-B 265 (1967), A207-A209.

4. W. Browder, (verbal communication).

5. S. Cappell, A splitting theorem for manifolds, Invent. Math 33 (1976), 69-170.

6. M. C. Crabb, $\mathbb{Z}_{2}$ Homotopy Theory, London Mathematical Society Lecture Note Series No. 44, Cambridge University Press, Cambridge (U.K.) and New York, 1980.

7. A. Dold, Partitions of unity in the theory of fibrations, Ann. of Math $\mathbf{7 8}$ (1963), 223-255.

8. K. H. Dovermann, $\mathbb{Z}_{2}$ surgery theory, Michigan Math.J. 28 (1981), 267-287.

9. K. H. Dovermann, M. Masuda, and R. Schultz, Conjugation involutions on homotopy complex projective spaces, Japanese J. Math 12 (1986), 1-34. 
10. K. H. Dovermann and R. Schultz, Equivariant surgery problems for certain manifolds with involution, preprint, Purdue University and University of Hawaii (1986).

11. J. Dupont, On the homotopy invariance of the tangent bundle I, Math. Scand. 26 (1970), 5-13.

12. On the homotopy invariance of the tangent bundle II, Math. Scand 16 (1970), 200-220.

13. F. Hirzebruch, Involutionen auf Mannigfaltigkeiten, Proceedings of the Conference on Transformation Groups (New Orleans, 1967), 148-166, Springer, Berlin-Heidelberg-New York, 1968.

14. I. James and E. Thomas, An approach to the enumeration problem for non-stable vector bundles, J. Math. Mech 14 (1965), 485-506.

15. T. Petrie and J. Randall, Transformation Groups on Manifolds 82 (1982), Marcel Dekker, New York.

16. M. Rothenberg, Torsion invariants and finite transformation groups, Proc. A. M. S. Sympos. Pure Math. 32 Pt. 1 (1978), 267-311.

17. R. Schultz, Homotopy decompositions of equivariant function spaces I, Math. Zeit. 131 (1973), 49-75.

18. Exotic spheres as stationary sets of homotopy sphere involutions, Michigan Math. J. 29 (1982), 121122.

19. S. H. Straus, Equivariant codimension one surgery, Ph. D. Dissertation, University of California, Berkeley, 1972 .

20. W. A. Sutherland, The fibre homotopy enumeration of nonstable sphere bundles and fibrings over real projective space, J. London Math. Soc. (2) 1 (1969), 693-704.

21. _ The Browder-Dupont invariant, Proc. London Math. Soc. (3) 33 (1976), 94-112.

22. _ Whitehead squares in Thom complexes, Proc. Edinburgh Math. Soc. 24 (1981), 221-229.

23. H. Toda, Composition Methods in Homotopy Groups of Spheres (1962), Princeton University Press, Princeton.

24. C. T. C. Wall, Surgery on Compact Manifolds, London Math. Soc. Monographs No. 1 Academic Press, London and New York (1970).

25. S. Waner, Equivariant fibrations and transfer, Trans. Amer. Math. Soc. 258 (1980), 369-384.

26. J. P. E. Hodgson, On spherical fibre spaces with $\mathbb{Z}_{2}$-actions, , preprint, Adelphi University, 1981(?).

27. K. H. Dovermann, Almost isovariant normal maps, preprint, University of Hawaii, 1986.

(Version 1.3: $\quad$ 10/27/86) 